\title{
TRANSFORMATION OF THE MEAN PLACE FROM FK4 TO FK5
}

\author{
Mitsuru Sôma and Shinko AoKI \\ National Astronomical Observatory \\ Mitaka, Tokyo 181, Japan
}

\begin{abstract}
Transformation procedures from the FK4 reference system of B1950.0 to the FK5 reference system of J2000.0 have been developed by Standish (1982) and by Aoki et al. (1983). We review here these procedures and discuss the differences between them. Especially we note that among researchers of this field a misunderstanding still exists in the problem at which stage the equinox correction should be applied. We show that the equinox correction should be applied in the precessing frame as Aoki et al. did. We also show that the epoch of the transfer from the FK4 to the FK5 in the transformation procedure is related to the systematic and individual corrections to the FK4.
\end{abstract}

\section{Introduction}

The matrix formulation of the transformation of the mean places and proper motions from the system of the FK4 at B1950.0 to that of the FK5 at J2000.0 has been developed by Standish (1982) and by Aoki et al. (1983). The main differences between these transformations are (1) in the application of the equinox correction and (2) in the epoch of the transfer from the FK4 system to the FK5 system.

Murray $(1989 \mathrm{a}, \mathrm{b})$ claims that the transformation by Standish is correct in both points mentioned above.

In this paper in Sect. 2 we discuss the matter of the equinox correction and show that the transformation by Aoki et al. is correct as for the application of the equinox correction. In Sect. 3 we deal with the matter of the epoch of the transfer and show that it is related to the systematic and individual corrections to the FK4.

Details about these matters will be published in a volume of Astronomy and Astrophysics.

\section{Application of the equinox correction}

In the Standish's or Murray's transformation the equinox correction is applied in the fixed frame whereas in the Aoki et al.'s transformation it is applied in the frame rotating by the precession. These transformation procedures are expressed as follows at the epoch of the transfer from the FK4 to the FK5: 
Standish or Murray (the epoch is B1950.0):

$$
\begin{aligned}
& \alpha^{N}=\alpha^{O}+E, \\
& \delta^{N}=\delta^{O}, \\
& \mu_{\alpha}^{N}+m^{N}+n^{N} \sin \left(\alpha^{O}+E\right) \tan \delta^{O}=\mu_{\alpha}^{O}+m^{O}+n^{O} \sin \left(\alpha^{O}+E\right) \tan \delta^{O}+\dot{E}, \\
& \mu_{\delta}^{N}+n^{N} \cos \left(\alpha^{O}+E\right)=\mu_{\delta}^{O}+n^{O} \cos \left(\alpha^{O}+E\right),
\end{aligned}
$$

Aoki et al. (the epoch is 1984 January 1.0):

$$
\begin{aligned}
& \alpha^{N}=\alpha^{O}+E, \\
& \delta^{N}=\delta^{O}, \\
& \mu_{\alpha}^{N}+m^{N}+n^{N} \sin \left(\alpha^{O}+E\right) \tan \delta^{O}=\mu_{\alpha}^{O}+m^{O}+n^{O} \sin \alpha^{O} \tan \delta^{O}+\dot{E}, \\
& \mu_{\delta}^{N}+n^{N} \cos \left(\alpha^{O}+E\right)=\mu_{\delta}^{O}+n^{O} \cos \alpha^{O},
\end{aligned}
$$

where $m$ and $n$ are the rates of the general precession in right ascension and declination based on the Newcomb's precession (superscript $O$ ) and the IAU 1976 precession (superscript $N$ ). Here the time unit is supposed to be the same in both sides of the equations for proper motions. The difference between Eqs. (1) and (2) is in the terms including $n^{O}$ in the equations for proper motions.

Murray $(1989 \mathrm{a}, \mathrm{b})$ considers the special case in which there is no change in the precession constant $\left(m^{N}=m^{O}, n^{N}=n^{O}\right)$, and the equinox correction is independent of time $(\dot{E}=0)$. He supposes that in this case both coordinate systems are inertial, in other words proper motions are the same in the two systems. The Eqs. (1c) and (1d) support his supposition while the Eqs. (2c) and (2d) don't, and he concludes that Standish's procedure is correct. Smith et al. (1989, in Note added in proof) and Yallop (1989) support this Murray's inference. But his inference is erroneous, because the precession is dependent on the location of the equinox (mean pole moves always toward the equinox at each instant) and therefore the equinox correction $E$ affects the obtained proper motions even if the equinox motion $\dot{E}$ is zero. Detailed considerations are given in the followings.

Proper motions of stars $\left(\mu_{\alpha}, \mu_{\delta}\right)$ are not directly determined from observations but are determined from the observed variations $(d \alpha / d t, d \delta / d t)$ of the mean places $(\alpha, \delta)$ from the following expressions:

$$
\begin{aligned}
& \frac{d \alpha}{d t}=m+n \sin \alpha \tan \delta+\mu_{\alpha} \\
& \frac{d \delta}{d t}=n \cos \alpha+\mu_{\delta} .
\end{aligned}
$$

The right ascension $\alpha$ appearing in the right sides of the above equations is, of cource, the observed right ascension in the catalog (FK4 or FK5) system and not in the dynamical system. Therefore if the catalog includes an error $E$ in the right ascension, it also affects the obtained proper motions. From this consideration the equations (2c) and (2d) are derived.

The correctness of the equations (2c) and (2d) can also be confirmed using the formulae of spherical triangles.

Thus we can conclude that as for the application of the equinox correction the procedure by Aoki et al. is correct. 
Murray insists that the equinox corrections determined from analyses of observations of the Sun, Moon and planets are corrections to the right ascension system of FK4 in the $B 1950.0$ frame, but this statemant is not correct. In fact, the equinox corrections so determined are corrections to the right ascension system of FK4 at the epoch of observation in the frame of date. For example, in the Eq. (5) given by Fricke (1985) and applied to observations, for example, by Hughes and Scott (1982):

$$
\begin{aligned}
\Delta \alpha= & -E+\cos \epsilon \sec ^{2} \delta \Delta L-\cos \alpha \tan \delta \Delta \epsilon \\
& +2 \sin \alpha \sec \delta \Delta h-2 \cos \alpha \sec \delta \cos \epsilon \Delta k,
\end{aligned}
$$

the quantity $E$ is the equinox correction at the epoch of observation in the frame of date. In the above equation $\Delta \alpha$ is the $O-C$ in the apparent right ascension of the Sun, $\epsilon$ is the obliquity of the ecliptic and $\Delta L$ is the correction to the mean longitude of the Sun.

It is obvious that Fricke (1982) assumed that the equinox correction $E(t)$ at any epoch in the frame of date can be expressed as

$$
E(t)=E_{1950}+\dot{E} t
$$

and obtained the values of $E_{1950}$ and $\dot{E}$ from analyses of observations of the Sun, Moon and planets. Because $\dot{E}$ in the above equation is theoretically equal to the value obtained from an analysis of FK4 proper motions in the frame of 1950.0, Fricke (1982) compared these values and obtained the final value of $\dot{E}$.

\section{Epoch of the transfer from the FK4 system to the FK5 system}

In the transformation by Aoki et al. the transfer from the FK4 system to the FK5 system is performed at the epoch of 1984 January 1 when the FK5 came into effect. Motivation of their transformation is that observed values such as stars' positions, UT1, etc. are not changed when the system of the catalog is changed, except stars' right ascensions and proper motions in right ascension, which are intentionally changed by the values obtained by Fricke (1982). Especially we note that their transformation is consistent with the new expression for the relationship between UT1 and GMST (Aoki et al., 1982). On the other hand Murray insists that the transfer should be performed at the epoch of B1950.0 as Standish did. The two $6 \times 6$ transformation matrices are given in Tables 1 and 2 . In calculating these matrices the application of the equinox correction is changed from Murray for the reasons mentioned in Sect. 2 and for the values of the equinox correction to the FK4 we adopt the values $E=0$ s.035 at J1950.0 and $\dot{E}=0$ s.085 / Julian century as Aoki et al. did in order to be consistent with the new expression for the relationship between UT1 and GMST. For the precession formulae based on the Newcomb's precession constant we use those given by Kinoshita (1975). The formulae for obtaining the position and velocity vectors from the mean place, proper motion, radial velocity and parallax, and vice versa, are given by Aoki et al.

The role of fundamental catalogs such as FK4 or FK5 is to define the equator and the equinox among stars on the celestial sphere at any epoch (see e.g. Woolard and 


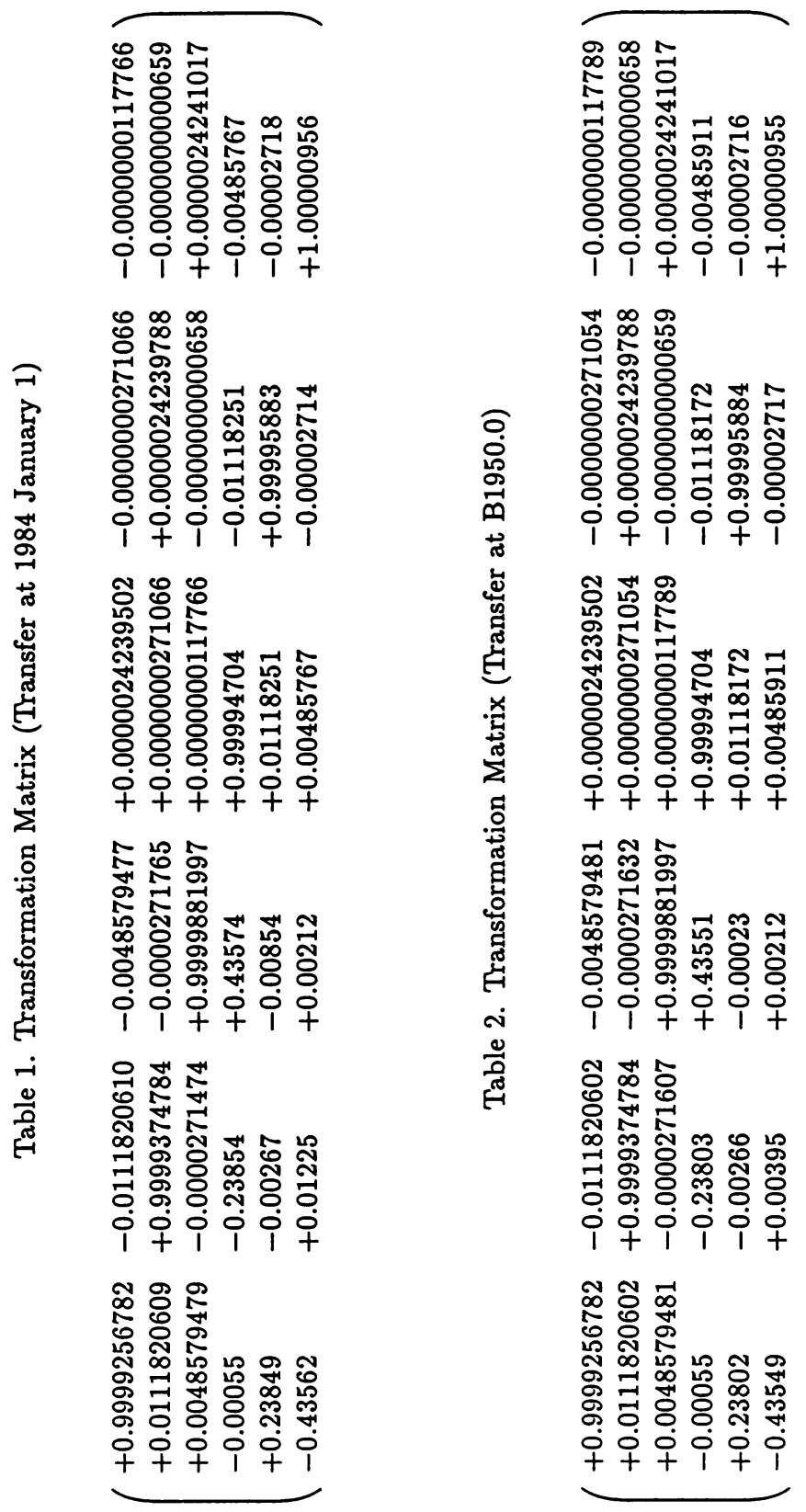


Clemence, 1966, pp. 376-377; or Fricke and Kopff, 1963, p.1). Whether the equator or the equinox defined by a fundamental catalog is coincident with the real equator or equinox is another question (the differences are called the systematic error of the catalog); absolute or fundamental observations will answer that question. In fact because we now know that the adopted precession constant in the FK4 has an error, if the equator defined by the FK4 is coincident with the real equator at some epoch, the equator defined by the FK4 at any other epoch is not coincident with the real equator. This fact suggests that the systematic error of the fundamental catalog depends on time. (The error of the first power of time can be eliminated by adjustment of proper motions, but the error of the second and higher power of time cannot be eliminated.) Besides systematic error there is an individual error for each star. This depends also on time. Murray implicitly assumes that the systematic and individual errors in the FK4 are zero at the epoch of B1950.0, but this cannot be justified, because positions and proper motions in the FK4 were not determined from observations only at the epoch of B1950.0.

If one uses the transformation matrix given in Table 1, one must apply the systematic correction to the FK4 system at the epoch of 1984 January 1, and if one uses the transformation matrix given in Table 2, one must apply the systematic correction to the FK4 system at the epoch of B1950.0. We can show that the matrix given in Table 2 can be derived from the matrix given in Table 1 by applying the systematic and individual corrections to the FK4 system.

If one ignores the systematic and individual corrections to the FK4 in the transformation to the FK5 system, the matrix given in Table 1 (Aoki et al.'s procedure) is recommended, because it is consistent with the definition of UT1. Also in the transformation of precise positions obtained by VLBI, the matrix given in Table 1 is recommended, because most of those observations are performed in the 1980's.

\section{References}

Aoki, S., Guinot, B., Kaplan, G. H., Kinoshita, H., McCarthy, D. D., Seidelmann, P. K., (1982), 'The new definition of Universal Time', Astron. Astrophys. 105, 359-361.

Aoki, S., Sôma, M., Kinoshita, H., Inoue, K. (1983) 'Conversion matrix of epoch B1950.0 FK4-based positions of stars to epoch J2000.0 positions in accordance with the new IAU resolutions', Astron. Astrophys. 128, 263-267.

Fricke, W. (1982) 'Determination of the equinox and equator of the FK5', Astron. Astrophys. 107, L13-L16.

Fricke, W. (1985) 'Fundamental Catalogues, past, present and future', Celes. Mech. 36, 207-239; or Veröff. Astron. Rechen-Inst., Heidelberg No. 31, Verlag G. Braun, Karlsruhe.

Fricke, W., Kopff, A. (1963), 'Fourth fundamental catalogue (FK4)', Veröff. Astron. Rechen-Inst., Heidelberg No. 10, Verlag G. Braun, Karlsruhe.

Hughes, J. A., Scott, D. K. (1982) 'Results of observations made with the six-inch transit circle 1963-1971', Publ. U. S. Naval Obs. Sec. Series Vol. XXIII, Part III.

Kinoshita, H. (1975) 'Formulas for Precession', SAO Special Report No. 364, Smithsonian Institution Astrophysical Observatory, Cambridge, Massachusetts. 
Murray, C. A. (1989a) 'The transformation of coordinates between the systems of B1950.0 and J2000.0, and the principal galactic axes referred to J2000.0', Astron. Astrophys. 218, 325-329.

Murray, C. A. (1989b) 'The transformation between the coordinate systems of FK4 at B1950.0 and FK5 at J2000.0', Highlights of Astronomy Vol. 8, pp. 482-483, Kluwer Academic Publishers, Dordrecht.

Smith, C. A., Kaplan, G. H., Hughes, J. A., Seidelmann, P. K., Yallop, B. D., Hohenkerk, C. Y. (1989) 'Mean and apparent place computations in the new IAU system. I. The transformation of astrometric catalog systems to the equinox J2000.0', Astron. J. 97, $265-273$.

Standish, E. M. Jr. (1982) 'Conversion of positions and proper motions from B1950.0 to the IAU system at J2000.0', Astron. Astrophys. 115, 20-22.

Woolard, E. W., Clemence, G. M. (1966), Spherical Astronomy, Academic Press Inc., New York.

Yallop, B. D. (1989) 'Apparent place reduction', Highlights of Astronomy vol. 8, pp. 481-482, Kluwer Academic Publishers, Dordrecht.

\section{Discussion}

SeIdelmann: The 1984 date is only an arbitrary date introducing the change. It is involved in the UT1 equation to maintain continuity across the change. It is not a part of the transformation of the star catalog mean position. It was not part of the determination of the equinox correction in the definition of the J2000 system of the FK5.

Sôma: The catalog positions have a close relation to the sidereal time and UT1. Therefore, it is important that the catalog positions should be determined in a consistent way with the equation for UT1 and sidereal time. But, as we have shown in this paper, the positions in the FK5 system do not depend on the epoch of transfer, if one applies the systematic correction to the FK4 properly.

MURRAY: In the first part of your paper you gave a fair description of the difference between us, but I still maintain that Fricke's motion of the equinox must be interpreted as being in the fixed frame B1950.

My motivation in entering into this subject was to determine the transformation to galactic coordinates for $\mathrm{J} 2000$. Using the transformations by Aoki et al., the galactic coordinates of an object calculated from its equatorial coordinates in the $\mathrm{J} 2000$ frame differ from those calculated from the B1950 frame, which is absurd!

Sôma: As shown in our paper, the equinox corrections must be applied in the moving frame. Aoki et al.'s transformation gives no inconsistency even in the galactic coordinates. 